\title{
Using Methods-Time Measurement to Connect Digital Humans and Motion Databases
}

\author{
Ali Keyvani ${ }^{1,3,4}$, Dan Lämkull ${ }^{2}$, Gunnar Bolmsjö ${ }^{3}$, and Roland Örtengren ${ }^{1}$ \\ ${ }^{1}$ Department of Product and Production Development, \\ Chalmers University of Technology, Gothenburg, Sweden \\ \{Ali.Keyvani, Roland. Ortengren\} @Chalmers.se \\ ${ }^{2}$ Virtual Methods \& IT, Volvo Car Corporation, Gothenburg, Sweden \\ Dan. Lamkull@volvocars.com \\ ${ }^{3}$ Department of Engineering Science, University West, Trollhättan, Sweden \\ Gunnar.Bolmsjo@hv.se \\ ${ }^{4}$ Innovatum AB, Trollhättan, Sweden
}

\begin{abstract}
To simulate human motions in DHM tools, using techniques which are based on real human data is one promising solution. We have presented a solution in this study to connect motion databases with DHM tools. We have showed that using a motion database with MTM-based annotations is a promising way in order to synthesize natural looking motions. A platform consists of a Motion Database, a Motion Generator, and a DHM tool was introduced and tested. The results showed successful application of the presented platform in the designed test case.
\end{abstract}

Keywords: Digital Human Modeling, Motion Databases, Human Motion Simulation.

\section{$1 \quad$ Introduction}

Digital Human Modeling (DHM) tools are getting more applicable in the simulation of production lines. However, there are still major challenges to generate natural looking motions of daily tasks when using existing commercial software packages [1]. In spite of the availability of various techniques for the modeling of human motion, the functionality of DHM tools is still limited in practice [2]. Based on success stories in the game and film industries, generating motion using techniques which are based on real human data is one promising solution [3,4]. Several challenges exist for using such data-driven techniques. There is a need for an extensive and properly structured motion database. Also, in order to compose a new motion based on the previously existing data, motions shall be suitably annotated in the database to be correctly searched and retrieved [5]. Finally, a connection between the DHM tool and Motion Database shall be established in a way that a requested scenario and retrieved motion can be communicated.

This study deals with these issues by using a motion database and Methods-Time Measurement (MTM) coding standard [6] to annotate a motion database. MTM is a 
recognized standard and was originally focused on analyzing and measuring methods for performing manual works and setting standard times in which the worker should complete a task. Besides for the regular application of MTM, there are a number of studies which have utilized MTM in generating/analyzing human-like motions. For example, the MTM system has been used to generate motions for humanoid robots [7], to analyze ergonomic workload [8,9], to simulate manual assembly sequence planning [10], and to generate motions for digital human models [11, 12].

Following these research works, this study highlights the feasibility to connect commercially available DHM tools to Motion Databases in a practical way. The objective of this work is to facilitate generation of natural looking motions. This is done by use of MTM vocabulary in the annotation of a Motion Database which is filled with captured human motions. In addition, a motion synthesizer module is developed. This module uses MTM vocabulary along with other information as communication objects between the user, Motion Database, and DHM tool in order to generate, search, and synthesize requested motion scenarios.

\section{Method}

Three main parts are involved in the motion generation process: Motion Database, Motion Generator, and DHM tool. These parts are interacting with each other in order to obtain desirable results. The Motion Database used in this study is a motion capture database presented by Keyvani et al. [13] and consists of several tables storing motions in a structured manner. The motion capture database supports different motion formats, multi skeleton configurations, and customized body zones (body zone is an optional collection of several joints recognized by a unique name e.g. right hand, upper body, etc.). Based on each skeleton configuration, these body zones are automatically matched with a reference joint table, and associated joints are then chosen. The Motion Database was filled with human motions which were gathered from different sources including Michigan Humosim laboratory [14], CMU Graphics Lab Motion Capture Database [15], and an in-house motion capture laboratory. A new set of annotation category based on MTM vocabulary is introduced to the database, and motions are annotated based on the new category. The Motion Database is expandable during time, and can be counted as an independent module as long as it uses the same annotating conventions and communicates by a standard language (SQL in this case).

One purpose of this study is providing the capability to support available DHM tools with solutions taken from real human motion. Therefore, generally any DHM tool which can import and export required data should work fine with this system. Most of the commercially available DHMs, such as Jack [16], support a scripting language which can provide a connection to the tool for data extraction. Finally, the motion generator part consists of number of modules including Task Builder user interface, Query Manager, Motion Synthesizer, Inverse Kinematic engine, and Internal Visualizer, which are presented in more detail in this section. 


\subsection{Annotating of Motion Database}

A new tagging category named MTM-Coding is introduced to the database schema. This category describes motions using a vocabulary based on the MTM-1 standard. The category consists of 20 main verbs (e.g. Reach, Move ...) and several sub-classes (called cases) defining the details of each basic motion. In the next step, the motions in the database are analyzed and annotated using the MTM coding. To do so, numbers of functions are implemented using Matlab to automatically detect the basic motions such as Reach, Move, and Turn. Manual adjustments were also needed in order to correct some of the wrong interpretations. For example, in a sample recorded motion, if the subject is transporting an object from one place to another, because no information is recorded in the motion files regarding the object, the system categorizes the motions as a 'Reach' instead of a 'Move', which has to be manually corrected. As a result, each motion in the database was divided into several motion segments, which were identified by different MTM codes. In general, three levels of tagging have been designed in the schema. Level I tags are the most general and apply to the whole motion (Table 1-Example 1). Level II tags are used to limit the scope of the tag spatially (Table 1-Example 2) or temporally, and level III tags are used to bound the tag domain both spatially and temporally (Table 1-Example 3). The MTM-Coding tags are mostly of a level III tag type, which means they bound the time (frame) range of the performed motion and also specify which joints are involved in each task. A number of advantages are gained by using the MTM system to categorize motions in the database:

- The MTM standard is well developed and provides a good level of detail.

- Multiple tags in parallel can be applied to a specific motion part.

- The assembly sheet instructions can be converted to the Task Builder format and vice versa, and the assembly sheet can be automatically generated after completion of the simulation.

- The process time can be calculated using the MTM standard.

Table 1. Structure of annotation table and examples of contents in the Motion Database

\begin{tabular}{|l||c|c|c|c|c|c|c|}
\hline Field Name & Tag Type & Tag Value & $\begin{array}{c}\text { Parent } \\
\text { Category }\end{array}$ & Motion ID & BodyZone & $\begin{array}{c}\text { Start } \\
\text { Frame }\end{array}$ & $\begin{array}{c}\text { End } \\
\text { Frame }\end{array}$ \\
\hline Field Type & String & String & Field & Integer & Field & Integer & Integer \\
\hline \hline Example 1 & Gender & Male & General & 2343 & - & - & - \\
\hline Example 2 & Experiment Type & Seated & Posture & 212 & Upper Body & - & - \\
\hline Example 3 & Reach & R_B & MTM & 212 & Right Arm & 100 & 147 \\
\hline
\end{tabular}

\subsection{Workflow}

The workflow of a sample process is shown in Fig. 1. First, the user creates a tasksequence by using MTM codes in the Task Builder user interface (1). Meanwhile, the Task Builder module receives necessary information, such as manikin current posture, 
objects positions and subject anthropometry (2). Next, this information is processed by the Query Manager (3) and resultant queries based on the required task and geometrical properties of the task (e.g. start point, end point, and joint constraints) are generated (4). Next, the Motion Database is searched and matching results are sent back to the Query Manager (5). Consequently, the motion Synthesizer module uses the resultant data from the Motion Database (6), along with the Inverse Kinematic Engine module (7), to synthesize a new motion which is tailored for the requested task-sequence. The user has the option to preview the result in the internal visualizer and fine tune it if necessary (8). Finally, the result is sent back to the DHM tool to be visualized and further analyzed (9).

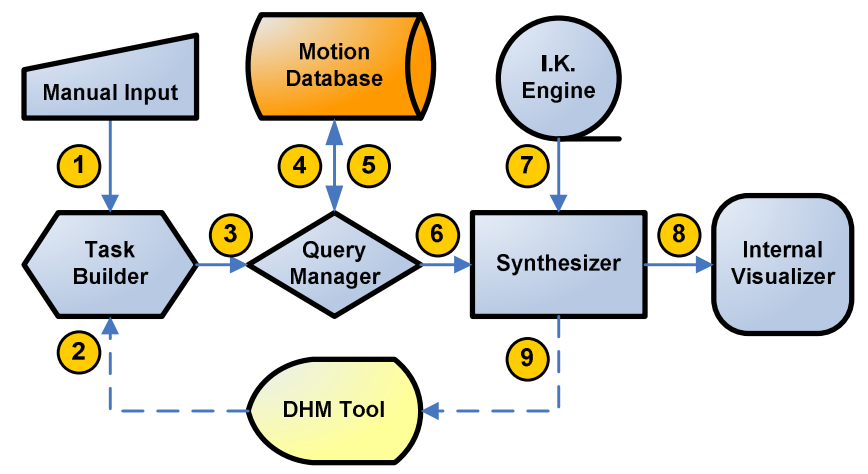

Fig. 1. Task-based motion generation workflow

\subsection{Query Manager}

A query manager is designed in the motion generator part to establish the communication between the database and the DHM tool. Required motions from the DHM tool are formulated using a sequence of MTM codes, along with motion specification (start point coordinates, end point coordinates, grip points, etc.) and subject anthropometry (stature, hand length, shoulder width, gender, etc.). The query manager searches the database for the closest matches, retrieves the results, and sends them to the motion synthesizer for any further necessary modifications. The query manager used in this study utilizes SQL language and a Matlab database toolbox to communicate with the Motion Database.

\subsection{Motion Synthesizer}

The motion synthesizer module consists of a set of functions implemented in Matlab, and enables the synthesis of a new motion based on segments of previously existing data in the database. Examples of these functions are cut, join, stretch, mix, blend, reposition, warp, etc.

It is likely that in many cases, an exact match would not be found among the existing motions stored in database. In such cases, the closest match is chosen and then a 'motion warping' technique similar to what is used by Witkin [17] is employed to 
reposition the end effector's positions. First, the new start/end point is resolved using an IK engine, and new joint angle values are determined. Then, obtained motion curves from the closest match are warped in order to match new start/end values. Eq.1 shows how the new values are calculated for each data point. For each data point $\theta(t)$ in time $t$ between control points $i$ and $i+1$ :

$$
\dot{\theta}(t)=\theta(t)+\left(1-\frac{t-t_{i}}{t_{i+1}-t_{i}}\right)^{n}\left(\theta_{i}-\theta(t)\right)+\left(\frac{t-t_{i}}{t_{i+1}-t_{i}}\right)^{n}\left(\theta_{i+1}-\theta(t)\right)
$$

When $t_{i}<t<t_{i+1}$

Where $\theta^{\prime}(t)$ is representing a new value for each data point in time $t$. $\theta_{i}$ and $\theta_{i+1}$ are values for control points and $n$ is a custom weighting factor ( $n=3$ is used).

\section{$3 \quad$ Validation}

A sample test case was designed and evaluated in order to validate the performance of the suggested solution. In this test case, we considered a scenario where a manikin (i) walks towards a table, (ii) squeezes between chair and table, (iii) sits on the chair, (iv) takes a part (bush) from a tray by right hand, (v) takes a part (pin) from a rack with left hand, (vi) inserts the pin in the bush's hole, and (vii) leaves the part on the table. Table 2 shows the MTM table of the test case while Table 3 shows the equivalent input generated by the user in the Task Builder. The current position of objects, grasp points, current posture, and subject's anthropometry were extracted from the external DHM tool. Generated query was sent to the Motion Database and the results were received back into Query Manager. To widen the search space and to consider solutions which can possibly fit after scaling the manikin, a relative measure of position over stature was used as search criteria. This means that for a subject with stature L, instead of searching for wrist position of $\mathrm{X}$, a measure of $\mathrm{X} / \mathrm{L}$ was searched and the matching result was scaled up/down if necessary. Also, for each specific task, different criteria were considered to sort the results for the closest match. As an example, for 'Reach' task, minimum deviation from requested start/end position was considered, or for 'Side_Step' task, closest stride length was considered. No 'Grasp' and 'Release' motions were available in the Motion Database, and therefore, no results were returned for those tasks. Simple grasp and release motions were manually generated and added to the database. In future developments, a hybrid method will be utilized to compensate these issues by mixing Database Motions with DHM generated motions to fill in the blanks. The 'Position' task is also simulated as 'Move'. In the next step, the final solution was tailored using motion synthesizer functions. Table 4 shows a summary of functions which are applied to different parts of the motion. Fig. 2. shows the layout of the designed test scenario and numbers of snapshots from the generated simulation. Roman numbers on each snapshot represent corresponding stage described at the beginning of this section. 
Table 2. MTM table of the test case is shown. LH and RH column represents left hand and right hand motions respectively, which are determined by MTM codes. The thick line around cells shows combined motions and they are executed together. The MTM codes which are used are as follows: W_F: walk forward, SS_C2: side step, SIT: stand to sit, R_B: reach by itself, G_1A: select grasp, M_B: move broad, T_S: turn, R_C: reach crowded, G_4B: grasp, M_C: move control, G_2: Regrasp, P3SE: position, RL1: release, R_E: reach ease

\begin{tabular}{|c|c|c|c|c|}
\hline Description & LH & Body & RH & Description \\
\hline $\begin{array}{r}\text { Walk to table front beside chair } \\
\text { Squeeze between table and chair } \\
\text { On chair } \\
\text { To pin in shelf B } \\
\text { To position Y } \\
\text { Orient the pin horizontally } \\
\text { Pin inside the bush hole }\end{array}$ & $\begin{array}{c}\text { R_C } \\
\text { G_4B } \\
\text { M_C } \\
\text { G_2 } \\
\text { P3SE } \\
\text { RL1 } \\
\text { R_E }\end{array}$ & $\begin{array}{c}\text { W_F } \\
\text { SS_C2 } \\
\text { SIT }\end{array}$ & $\begin{array}{l}\text { R_B } \\
\text { G_1A } \\
\text { M_B } \\
\text { T_S }\end{array}$ & $\begin{array}{l}\text { To bush in tray A } \\
\text { To position X } \\
\text { Reorient the bush to } \\
\text { keep hole horizontal }\end{array}$ \\
\hline
\end{tabular}

Table 3. Task Builder table created by the user. The values for parameters are automatically decided by the DHM tool and transferred to the task builder module. BodyZones and Order are determined by the user. Equal numbers in order column mean simultaneous execution of tasks. Right/left hand means wrist joint in addition to finger joints. Right/left arm means shoulder joints in addition to elbow and wrist joints. Right/left chain means all joints starting from the base of body, spine, thorax, and right/left arm.

\begin{tabular}{|r|lllll|}
\hline MTM Task & Type & Order & BodyZone & Par1 & Par2 \\
\hline Walk & W_F & 1 & LowerBody & Loc1 & Loc2 \\
Side Step & SS_C2 & 2 & LowerBody & Loc2 & Loc3 \\
SIT & SIT & 3 & FullBody & & \\
Reach & R_B & 4 & RightChain & RWrist Current Loc & Loc4 \\
Grasp & G_1A & 5 & RightHand & & \\
Move & M_B & 6 & RightHand & Loc4 & Loc5 \\
Turn & T_S & 6 & RightHand & CurrentValue & Coord1 \\
Reach & R_C & 7 & LeftChain & LWrist Current Loc & Loc6 \\
Grasp & G_4B & 8 & LeftHand & & \\
Move & M_C & 9 & LeftChain & Loc6 & Loc7 \\
Grasp & G_2 & 9 & LeftHand & Coord2 & \\
Position & P3SE & 10 & LeftArm & Path1 & \\
Release & RL1 & 11 & RightHand & & \\
Release & RL1 & 11 & LeftHand & & \\
Reach & R_E & 12 & RightChain & Loc5 & \\
Reach & R_E & 12 & LeftChain & Loc7 & \\
\hline
\end{tabular}


Table 4. Summary of functions which are used during motion synthesis

\begin{tabular}{l|lr}
\hline $\begin{array}{l}\text { Applied } \\
\text { Function }\end{array}$ & Description & Example Task \\
\hline Reposition & To shift a motion in order to match start position & Side Step (S.S) \\
Join & To join motion pieces together & Walk+S.S. \\
Warp & To change start end point of a motion & Reach \\
Blend & To smooth transition & Walk+S.S.+SIT \\
Reconfigure & To match different skeletons & - \\
Cut & To match start/end point & Move \\
Stretch & To scale the time of a process & Turn \\
\hline
\end{tabular}

\section{Discussion}

We have presented a solution in this study to connect motion databases with DHM tools. We have showed that using a motion database with MTM-based annotations is a promising way in order to synthesize natural looking motions. A platform consists of a Motion Database, a Motion Generator, and a DHM tool was introduced and tested. The results showed successful application of the presented platform in the designed test case.

We have noticed that when tailoring a new motion scenario there is a need to integrate data-driven methods, like what is presented in this study, with inverse kinematic techniques. This is especially important when a sufficiently close match in the database is missing, or when a complicated path needs to be followed. Moreover, using more smart methods to formulate the motion patterns and to extrapolate existing data for similar cases (re-targeting, re-subjecting) are desirable for such integration in the future.

At the moment, the user needs to define the tasks in a low-level language and assign the corresponding BodyZones manually to each task. In future work, a rule-based system will be substituted, which provides the capability to define tasks in a highlevel language form. The low-level tasks and corresponding BodyZones are then inferred and assigned automatically.

We have observed that blending of motions pieces together and warping of motioncurves can increase the smoothness of the final motion, and therefore, the realistic look of it. A simple linear method has been used in this study just for evaluation purposes, while more sophisticated techniques which have been studied by other researchers such as Kovar [18], Ménardais [19], and Park [20] can improve the functionality of the system in the future.

In general when using motion capture a limited amount of persons is captured, thus it is often not tenable to claim that the motion behavior is valid for a larger group of people. To try to find a solution of this problem, several DHM-tool providers have developed scaling functions, thus it is possible to use a subject of any stature and scale the subject, such that it appears to match the anthropometry that is desired for testing. Currently, there is no data to support the assumption that this practice produces the same results as would be obtained using a subject who is actually of the desired size $[21,22]$. 
The performance of a task is often limited by the ability to maintain balance [23]. According to Lockett et al. [24], body balance control is one feature that is required to properly and realistically simulate human activity. We have assumed that motions which are stored in the database have maintained balance in the timed of recording. However, one limitation to this assumption is that we have neglected the changes in balancing when mixing segments of motions from different parts of the body.

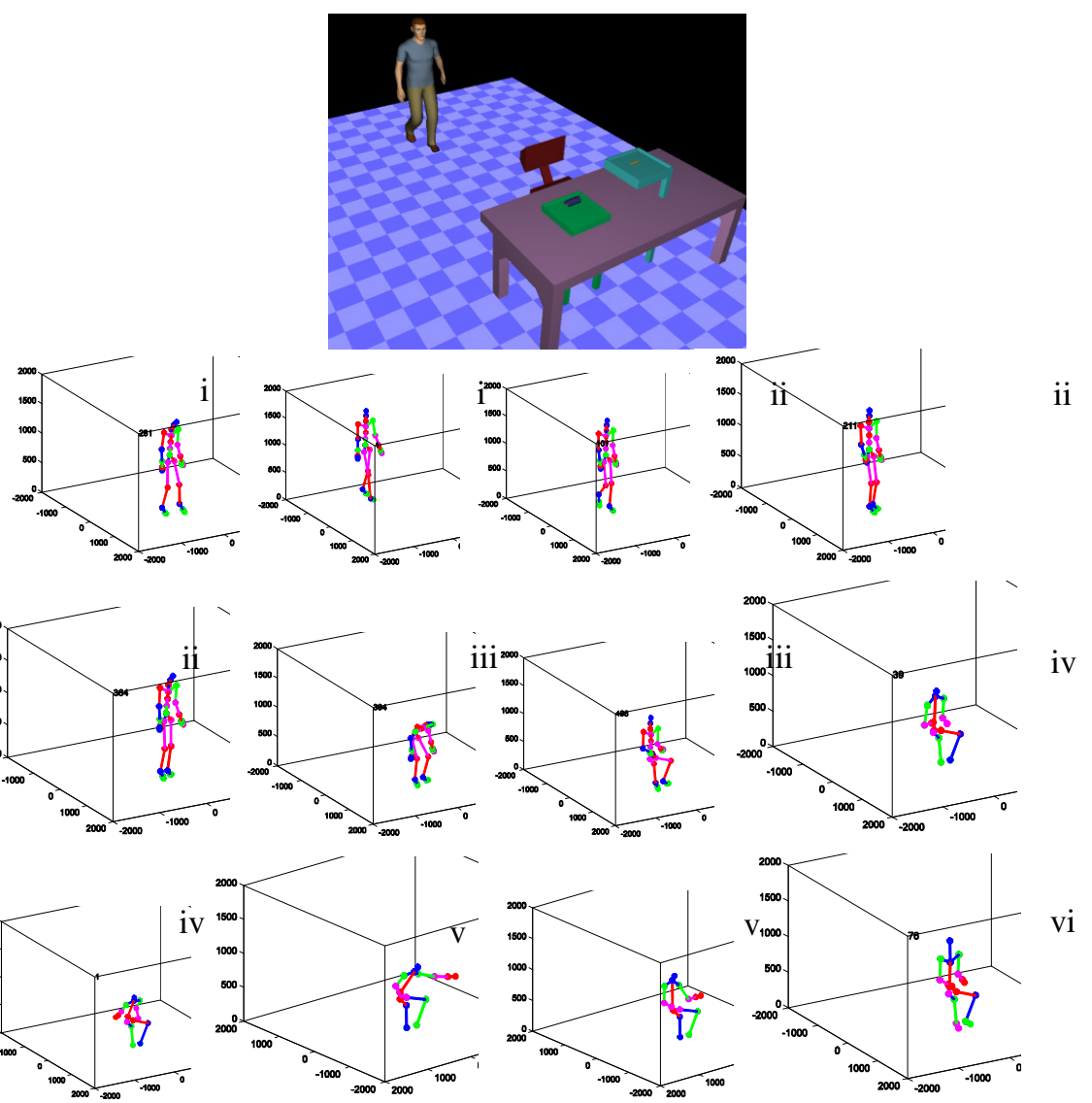

Fig. 2. Up: Layout of the test scenario, Down: Snapshots from the resulted simulation

Acknowledgment. Part of the data used in this project was obtained from mocap.cs.cmu.edu. This data was created with funding from NSF EIA-0196217. Another part of the data was received from Humosim laboratory at the University of Michigan. Access to these data provided valuable insight for the progress of this project, and usage of them in this study is acknowledged by the authors. 


\section{References}

1. Lämkull, D., Hanson, L., Örtengren, R.: A comparative study of digital human modelling simulation results and their outcomes in reality: A case study within manual assembly of automobiles. Int. J. Ind. Ergonom. 39, 428-441 (2009)

2. Chaffin, D.B.: Improving digital human modelling for proactive ergonomics in design. Ergonomics 48, 478-491 (2005)

3. Zordan, V.B., Hodgins, J.K.: Motion capture-driven simulations that hit and react. In: Proceedings of the 2002 ACM SIGGRAPH/Eurographics Symposium on Computer Animation, pp. 89-96, 545276. ACM (2002)

4. Yamane, K., Kuffner, J.J., Hodgins, J.K.: Synthesizing animations of human manipulation tasks. ACM Trans. Graph. 23, 532-539 (2004)

5. Barbi, J., Safonova, A., Pan, J.-Y., Faloutsos, C., Hodgins, J.K., Pollard, N.S.: Segmenting motion capture data into distinct behaviors. In: Proceedings of Graphics Interface 2004, pp. 185-194. Canadian Human-Computer Communications Society, London (2004)

6. Karger, D.W., Bayha, F.H.: Engineered work measurement: the principles, techniques, and data of methods-time measurement background. Industrial Press, New York (1987)

7. Drumwright, E., Ng-Thow-Hing, V., Mataric, M.: Toward a vocabulary of primitive task programs for humanoid robots. In: International Conference on Development and Learning, ICDL (2006)

8. Laring, J., Forsman, M., Kadefors, R., Örtengren, R.: MTM-based ergonomic workload analysis. Int. J. Ind. Ergonom. 30, 135-148 (2002)

9. Christmansson, M., Falck, A.C., Amprazis, J., Forsman, M., Rasmusson, L., Kadefors, R.: Modified method time measurements for ergonomic planning of production systems in the manufacturing industry. Int. J. Prod. Res. 38, 4051-4059 (2000)

10. Kanai, S., Takahashi, H., Makino, H.: ASPEN: computer-aided assembly sequence planning and evaluation system based on predetermined time standard. CIRP AnnalsManufacturing Technology 45, 35-39 (1996)

11. Kuo, C.F., Wang, M.J.: Motion generation from MTM semantics. Comput. Ind. 60, 339348 (2009)

12. Kuo, C.-F., Wang, M.-J.J.: Motion generation and virtual simulation in a digital environment. Int. J. Prod. Res. 50, 6519-6529 (2012)

13. Keyvani, A., Johansson, H., Ericsson, M., Lämkull, D., Örtengren, R.: Schema for Motion Capture Data Management. In: Duffy, V.G. (ed.) ICDHM 2011. LNCS, vol. 6777, pp. 99-108. Springer, Heidelberg (2011)

14. Reed, M.P., Faraway, J., Chaffin, D.B., Martin, B.J.: The HUMOSIM Ergonomics Framework: A new approach to digital human simulation for ergonomic analysis. SAE Technical Paper 01-2365 (2006)

15. Carnegie Mellon University's Graphics Lab, http://mocap. cs . cmu . edu

16. Siemens Product Lifecycle Management Software Inc., http://www.plm.automation.siemens.com/en_us/products / tecnomatix/assembly_planning/jack/index.shtml

17. Witkin, A., Popovic, Z.: Motion warping. In: Proceedings of the 22nd Annual Conference on Computer Graphics and Interactive Techniques, pp. 105-108. ACM (1995)

18. Kovar, L., Gleicher, M.: Flexible automatic motion blending with registration curves. In: Proceedings of the 2003 ACM SIGGRAPH/Eurographics Symposium on Computer Animation, pp. 214-224. Eurographics Association, San Diego (2003) 
19. Ménardais, S., Multon, F., Kulpa, R., Arnaldi, B.: Motion blending for real-time animation while accounting for the environment. In: Proceedings of Computer Graphics International, pp. 156-159. IEEE (2004)

20. Park, S.I., Shin, H.J., Kim, T.H., Shin, S.Y.: On-line motion blending for real-time locomotion generation. Comput. Animat/. Virt. W. 15, 125-138 (2004)

21. Godin, C., Chiang, J., Stephens, A., Potvin, J.: Assessing the accuracy of ergonomic analyses when human anthropometry is scaled in a virtual environment. SAE Technical Paper 01-2319 (2006)

22. Johansson, I., Larsson, M.: Evaluation of the manikin building function in eM-RAMSIS when using Motion Capture. Department of Human Work Sciences. Luleå University of Technology (2007)

23. Parkinson, M.B., Reed, M.P.: Considering driver balance capability in truck shifter design. SAE Technical Paper 01-2360 (2006)

24. Lockett, J.F., Assmann, E., Green, R., Reed, M.P., Raschke, U., Verriest, J.-P.: Digital human modeling research and development user needs panel. SAE Transactions 114, 886-890 (2005) 